\title{
PAPER
}

\section{White matter damage in Alzheimer's disease assessed in vivo using diffusion tensor magnetic resonance imaging}

\author{
M Bozzali, A Falini, M Franceschi, M Cercignani, M Zuffi, G Scotti, G Comi, M Filippi
}

J Neurol Neurosurg Psychiatry 2002;72:742-746

\begin{abstract}
See end of article for authors' affiliations

.....................

Correspondence to: Dr M Filippi, Neuroimaging Research Unit, Department of Neuroscience, Scientific Institute and University Ospedale San Raffaele, Via Olgettina 60, 20132 Milan, Italy; m.filippi@hsr.it

Received 10 August 2001 In revised form

7 November 2001

Accepted 21 November 2001
\end{abstract}

\begin{abstract}
Objective: To investigate the extent and the nature of white matter tissue damage of patients with Alzheimer's disease using diffusion tensor magnetic resonance imaging (DT-MRI).

Background: Although Alzheimer's disease pathology mainly affects cortical grey matter, previous pathological and MRI studies showed that also the brain white matter of patients is damaged. However, the nature of Alzheimer's disease associated white matter damage is still unclear.

Methods: Conventional and DT-MRI scans were obtained from 16 patients with Alzheimer's disease and 10 sex and age matched healthy volunteers. The mean diffusivity (D)), fractional anisotropy (FA), and inter-voxel coherence $(C)$ of several white matter regions were measured.

Results: $\bar{D}$ was higher and FA lower in the corpus callosum, as well as in the white matter of the frontal, temporal, and parietal lobes from patients with Alzheimer's disease than in the corresponding regions from healthy controls. $\bar{D}$ and FA of the white matter of the occipital lobe and internal capsule were not different between patients and controls. $C$ values were also not different between patients and controls for any of the regions studied. Strong correlations were found between the mini mental state examination score and the average overall white matter $\bar{D}(r=0.92, p<0.001)$ and FA $(r=0.78$; $\mathrm{p}<0.001)$.

Conclusions: White matter changes in patients with Alzheimer's disease are likely to be secondary to wallerian degeneration of fibre tracts due to neuronal loss in cortical associative areas.
\end{abstract}

$\mathrm{T}$ he loss of layer III and V large pyramidal neurons, particularly in cortical association regions, is the pathological substrate of the progressive dementing process in Alzheimer's disease. ${ }^{1-3}$ Consistent with this, MRI studies ${ }^{3-7}$ have shown overall brain atrophy or atrophy of specific brain structures in patients with Alzheimer's disease when compared with non-demented elderly controls. More recently, MR based techniques have been applied to the study of patients with Alzheimer's disease to achieve accurate in vivo estimates of Alzheimer's disease related metabolic ${ }^{89}$ or microscopic structural changes ${ }^{10-16}$. In detail, diffusion weighted MRI (DWMRI) studies have shown that in patients with Alzheimer's disease there is a considerable amount of microscopic white matter pathology. ${ }^{10-14}{ }^{16}$ Although this finding fits well with previous neuropathological observations, ${ }^{17}{ }^{18}$ the extent and the nature of microscopic white matter damage in Alzheimer's disease has not been fully investigated yet.

DW-MRI provides a unique form of MR contrast that enables the diffusional motion of water molecules to be measured and, as a consequence of the interactions between tissue water and cellular structures, provides information about the size, shape, orientation, and geometry of brain structures. ${ }^{19}$ Cellular structures in the CNS restrict water molecular motion, and so pathological processes that modify tissue integrity, thus removing some of the "restricting" barriers can result in an increased diffusion coefficient. Because some cellular structures such as axons are aligned on the scale of an image voxel, the restriction to diffusion is also dependent on the direction in which diffusion is measured. As a consequence, to provide a full in vivo characterisation of diffusion, diffusion tensor MR imaging (DT-MRI ${ }^{20}$ was developed. From the tensor, it is possible to derive the mean diffusivity $(\bar{D}=1 / 3$ of the trace of the tensor) and the fractional anisotropy (FA), which is one of the most robust measures of anisotropy, ${ }^{21}$ and which measures the degree of deviation from isotropic diffusion in individual voxels. More recently, an additional DT-MRI derived index has been proposed. ${ }^{22}$ This index measures the degree of similarity of orientation of neighbouring voxels and it is named intervoxel coherence $(C) .^{22}$

Previous DW-MRI studies of white matter changes in patients with Alzheimer's disease were limited by the use of pulse sequences sensitive to motion artifacts, as well as by the calculation of rotationally variant indices of diffusion. ${ }^{10-12} 1415$ Two recent preliminary DT-MRI studies were also limited by the fact that in one study ${ }^{13}$ only tissue anisotropy of a few white matter regions was measured and in the other ${ }^{16}$ regional white matter changes were not assessed. In the present study, we used DT-MRI to its full advantage (we calculated D, FA, and C) to investigate the extent of tissue damage of several brain white matter regions in patients with Alzheimer's disease. The ultimate goals of the study were to provide a complete picture of the distribution of microstructural white matter damage in Alzheimer's disease and to improve our understanding of their nature.

\section{PATIENTS AND METHODS \\ Patients}

Sixteen patients (six women and 10 men, mean age 69.6 years, range 64-85 years; median disease duration 24 months, range 12-36 months) who met the National Institute of Neurological and Communicative Disease and Stroke (NINCDS) and the Alzheimer's Disease and Related Disorders Association (ADRDA) criteria $^{23}$ for a diagnosis of clinically probable Alzheimer's disease were recruited from patients attending a specialist dementia clinic. Ten sex and age matched healthy controls (four women and six men, mean age 66.1 years, range 61-75 years-the age difference between controls and patients

Abbreviations: DT-MRI, diffusion tensor magnetic resonance imaging; $\bar{D}$, mean diffusivity; FA, fractional anisotropy; $C$, intervoxel coherence; MMSE, mini mental state examination; TSE, turbo spin echo; PGSE, pulsed gradient spin echo; ROls, regions of interest 
with Alzheimer's disease was not statistically significant) were recruited. The controls had no complaints of cognitive problems and no evidence of cognitive deficits on formal testing. Major systemic, psychiatric, and other neurological illnesses were carefully investigated and excluded in all subjects. To minimise the inclusion of patients with Alzheimer's disease and associated major ischaemic vascular damage (which is also known to cause cognitive decline), three patients were excluded from the study as they had either one hyperintense area with a diameter equal or greater than 5 $\mathrm{mm}$ or more than four hyperintense areas smaller than $5 \mathrm{~mm}$ in diameter on T2 weighted MRI. Local ethics committee approval and written informed consent from all the patients were obtained before study initiation.

\section{Neuropsychological assessment}

To obtain an overall assessment of cognitive function each subject was administered the mini mental state examination $(\text { MMSE })^{24}$ by a trained neuropsychologist, unaware of the MRI results, within 48 hours from acquisition of the MR images. Median MMSE score (corrected for age and the level of education) was 19.4 (range 9.3-25.4) for patients with Alzheimer's disease and 28.6 (range 26.8-30.7) for control subjects. ${ }^{25}$

\section{MRI acquisition}

Brain MR scans were obtained on a magnet operating at $1.5 \mathrm{~T}$. On a single occasion, images using the following pulse sequences were obtained from all subjects without moving them from the scanner:(a) dual echo turbo spin echo (TSE) $(\mathrm{TR}=3300 \mathrm{~ms}, \mathrm{TE}=16 / 98 \mathrm{~ms}$, echo train length $=5) ;(b) \mathrm{Tl}$ weighted SE (TR=650 ms, TE $=12 \mathrm{~ms}) ;(c)$ pulsed gradient spin echo (PGSE) echo planar pulse sequence (interecho spacing $=0.8 \mathrm{~ms}, \mathrm{TE}=123 \mathrm{~ms}$ ), with diffusion gradients applied in eight non-collinear directions, chosen to cover three dimensional space uniformly. ${ }^{26}$ The duration and maximum amplitude of the diffusion gradients were respectively $25 \mathrm{~ms}$ and $21 \mathrm{mTm}^{-1}$, giving a maximum $\mathrm{b}$ factor in each direction of $1044 \mathrm{~s} \mathrm{~mm}^{-2}$. To optimise the measurement of diffusion only two $b$ factors were used ${ }^{27}\left(b_{1} \approx 0, b_{2}=1044 \mathrm{~s} \mathrm{~mm}^{-2}\right)$. Fat saturation was performed using a four radio frequency binomial pulse train to avoid chemical shift artifact. A birdcage head coil of $\sim 300 \mathrm{~mm}$ diameter was used for signal transmission and for reception. For the dual echo and Tl weighted sequences, 24 contiguous interleaved axial slices were acquired with $5 \mathrm{~mm}$ slice thickness, 256×256 matrix and $250 \times 250 \mathrm{~mm}$ field of view. The slices were positioned to run parallel to a line that joins the most inferoanterior and inferoposterior parts of the corpus callosum. For the PGSE scans, 10 $5 \mathrm{~mm}$ thick slices were acquired, with the same orientation of the dual echo scans, positioning the second last caudal slice to match exactly the central slices of the other image sets. This set of slices was chosen as these central slices are less affected by the distortions due to $\mathrm{B}_{0}$ field inhomogeneity, which can affect image coregistration. Also, this set of slices allowed us to cover a relatively large portion of the cerebral hemispheres where white matter is highly represented. PGSE images had a $128 \times 128$ matrix and a $250 \times 250 \mathrm{~mm}$ field of view.

\section{MRI analysis and postprocessing}

Two experienced observers, unaware to whom the scans belonged, identified by consensus any white matter hyperintense area on the dual echo scans from all subjects. Brain volumes were measured by one observer, again unaware to whom the scans belonged, using a seed growing segmentation technique based on signal intensity thresholding and characterised by high intrarater reproducibility ${ }^{28}$ After transferral of all images to a workstation (Sun Sparcstation; Sun Microsystem, Mountain View, CA, USA), PGSE images were first corrected for geometrical distortion induced by eddy currents using an algorithm which maximises mutual information between the diffusion unweighted and weighted images. ${ }^{29}$ Then the PGSE images were interpolated to the same matrix size as the dual echo and the $b=0$ step of the PGSE images was coregistered with the long echo images of the dual echo scans using a two dimensional third order warping function, provided by the AIR package..$^{30}$ The same transformation parameters were also used to map the EP images on the spatial coordinates of the dual echo. Then, assuming a monoexponential relation between signal intensity and the product of the b matrix and diffusion tensor matrix components, the tensor was estimated statistically, using a multivariate linear regression model. ${ }^{31} \overline{\mathrm{D}}$ and FA maps were derived as previously described. ${ }^{32} \mathrm{C}$ maps were produced, as described by Pfefferbaum et al, ${ }^{22}$ by calculating the average angle between the eigenvector of the largest eigenvalue (whose direction reflects fibre orientation) of a given voxel and its neighbours, using a $3 \times 3$ symmetric kernel. $\bar{D}, \mathrm{FA}$, and $\mathrm{C}$ were measured in different regions of the white matter, which were carefully selected on the dual echo scans to avoid partial volume averaging from the CSF. Rectangular regions of interest (ROIs) of variable size (range $=11.4-46.7$ $\mathrm{mm}^{2}$ ), depending on the anatomical region studied, were placed bilaterally in the white matter of the following areas: the genu and the splenium of the corpus callosum, the posterior limb and the genu of the internal capsule, anterior and posterior pericallosal areas, and the white matter of the four cerebral lobes. The genu and the splenium of the corpus callosum were sampled on the three consecutive slices on which they were fully volumed. Anterior and posterior pericallosal ROIs were positioned laterally to the splenium and the genu of the corpus callosum in the same three slices. The posterior limb and the genu of the internal capsule, respectively indexed as the regions bounded by the corner between the head of the caudate nucleus and the pallidum and by the pallidum and the thalamus, were marked on two contiguous slices. Frontal lobe white matter was sampled on three contiguous slices starting from the most cranial slice which included a fully volumed lateral ventricle in at least one hemisphere. Parietal lobe ROIs were positioned in the white matter posterior to the central sulcus on the most caudal slice where it was visible and on the subsequent more cranial slice. Temporal lobe white matter was sampled on three contigous slices and the ROIs were placed posterolaterally to the lateral fissure, starting from the most caudal slice on which it was present. The occipital lobe ROIs were placed within the optic radiations on two contiguous slices, starting from the most caudal slice on which the occipital horn of the lateral ventricle was imaged. Figure 1 illustrates the location of all the white matter ROIs. The ROIs were transferred onto $\overline{\mathrm{D}}, \mathrm{FA}$, and $\mathrm{C}$ maps for each subject and average $\bar{D}, \mathrm{FA}$, and $\mathrm{C}$ were calculated for each of the selected white matter areas and for the overall white matter.

\section{Statistical analysis}

A Student's $t$ test for non-paired data was used to compare $\overline{\mathrm{D}}$, $\mathrm{FA}$, and $\mathrm{C}$ values of the white matter from patients with Alzheimer's disease and controls. Because eight comparisons were performed for each of the considered DT-MRI quantities, only Bonferroni corrected $\mathrm{p}$ values $\leqslant 0.006$ were considered as statistically significant. The correlations between average $\overline{\mathrm{D}}$, FA, and $C$ values of the overall white matter studied and the MMSE scores were investigated using Spearman's rank correlation coefficient.

\section{RESULTS}

Aspecific white matter hyperintense lesions were detected on scans from four controls and five patients with Alzheimer's disease. By definition, these lesions were no more than four in each patient and smaller than $5 \mathrm{~mm}$ in diameter. As expected, brain volume was lower in patients with Alzheimer's disease 


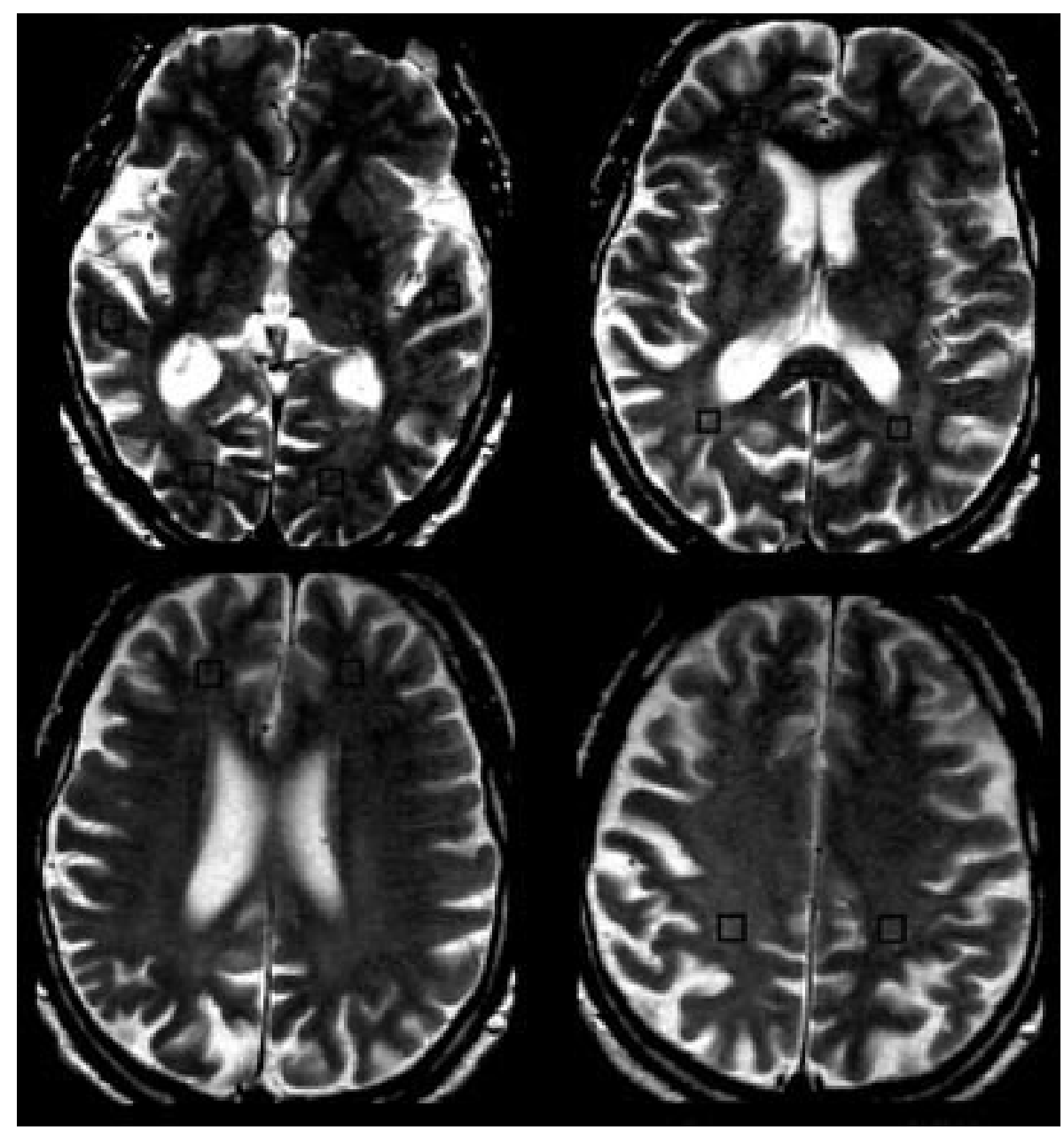

Figure 1 Location of the white matter regions of interest in a patient with Alzheimer's disease. (A) genu and posterior limb of the internal capsule, and white matter of the temporal and occipital lobes; (B) genu and splenium of the corpus callosum, and anterior and posterior pericallosal areas; (C) white matter of the frontal lobes; (D) white matter of the parietal lobes.

than in controls (mean brain volumes were $918 \mathrm{ml}$ ( SD $82 \mathrm{ml}$ ) and $1067 \mathrm{ml}$ (SD $99 \mathrm{ml}$ ), respectively; $\mathrm{p}=0.003$ ).

$\overline{\mathrm{D}}$ was significantly higher and FA significantly lower in the corpus callosum, as well as in the white matter of the frontal, temporal, and parietal lobes from patients with Alzheimer's disease than in the corresponding regions from healthy controls (tables 1 and 2). D and FA of the white matter of the occipital lobe, internal capsule and pericallosal areas were not different between patients and controls. C values were also not

Table 1 Mean (SD) $\bar{D}$ values of the selected white matter areas from $A D$ patients and controls

\begin{tabular}{llll}
\hline & AD & Controls & p Value* \\
\hline Corpus callosum & $1.04(0.10)$ & $0.86(0.05)$ & $<0.001$ \\
Pericallosal areas & $0.93(0.1)$ & $0.84(0.04)$ & 0.008 \\
Frontal lobe & $0.93(0.11)$ & $0.80(0.05)$ & $<0.001$ \\
Temporal lobe & $1.07(0.24)$ & $0.86(0.06)$ & 0.003 \\
Parietal lobe & $0.93(0.10)$ & $0.82(0.05)$ & 0.004 \\
Occipital lobe & $0.93(0.10)$ & $0.93(0.09)$ & 0.99 \\
Internal capsule & $0.81(0.07)$ & $0.81(0.04)$ & 0.88 \\
All white matter areas & $0.94(0.07)$ & $0.86(0.03)$ & $<0.001$ \\
\hline
\end{tabular}

* Student's $t$ test for non-paired data

$\overline{\mathrm{D}}$, mean diffusivity, expressed in units of $\mathrm{m}^{2} \mathrm{~s}^{-1} \times 10^{-9}$. Corpus callosum $\bar{D}$ values were obtained by averaging the values of the splenium and genu of the corpus callosum. Pericallosal $\bar{D}$ values were obtained by averaging the values of the anterior and posterior pericallosal areas. Internal capsule $\bar{D}$ values were obtained by averaging the values of genu and posterior limb of this structure. different between patients with Alzheimer's disease and controls for any of the regions studied (table 3 ).

Strong correlations were found between the MMSE score and the average overall white matter $\overline{\mathrm{D}}$ and FA (fig 2). The $r$ value of the correlation between average white matter $\mathrm{C}$ and MMSE score was $0.52(\mathrm{p}=0.06)$.

\section{DISCUSSION}

Pathoanatomical ${ }^{17}$ and $\mathrm{MRI}^{33-35}$ studies have shown macroscopic white matter lesions in patients with Alzheimer's

Table 2 Mean (SD) FA values of the selected white matter areas from $A D$ patients and controls

\begin{tabular}{llll}
\hline & AD & Controls & p Value* \\
\hline Corpus callosum & $0.18(0.03)$ & $0.22(0.02)$ & 0.001 \\
Pericallosal areas & $0.29(0.08)$ & $0.36(0.03)$ & 0.01 \\
Frontal lobe & $0.21(0.03)$ & $0.29(0.06)$ & $<0.001$ \\
Temporal lobe & $0.17(0.04)$ & $0.28(0.07)$ & $<0.001$ \\
Parietal lobe & $0.24(0.06)$ & $0.33(0.03)$ & $<0.001$ \\
Occipital lobe & $0.22(0.03)$ & $0.22(0.06)$ & 0.88 \\
Internal capsule & $0.43(0.05)$ & $0.44(0.04)$ & 0.95 \\
All white matter areas & $0.32(0.04)$ & $0.37(0.02)$ & 0.001 \\
\hline
\end{tabular}

*Student's $t$ test for non-paired data; FA, fractional anisotropy. Corpus callosum FA values were obtained by averaging the values of the splenium and genu of the corpus callosum. Pericallosal FA values were obtained by averaging the values of the anterior and posterior pericallosal areas. Internal capsule FA values were obtained by averaging the values of genu and posterior limb of this structure. 

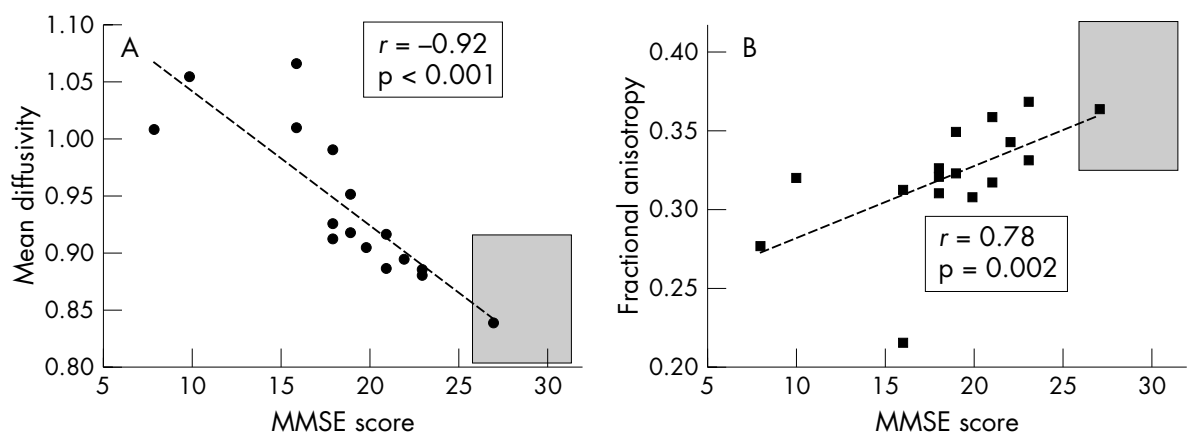

Figure 2 Scatterplots of the white matter mean diffusivity (expressed in units of $\left.\mathrm{m}^{2} \mathrm{~s}^{-1} \times 10^{-9}\right)(\mathrm{A})$, and fractional anisotropy (B) values versus mini mental state scale (MMSE) scores in patients with Alzheimer's disease. The grey rectangles represent the mean values $( \pm 2$ SD) for mean diffusivity and MMSE (A) and for fractional anisotropy and MMSE (B) from the normal controls.

Table 3 Mean (SD) C values of the selected white matter areas from $A D$ patients and controls

\begin{tabular}{llll}
\hline & AD & Controls & p Value* \\
\hline Corpus callosum & $0.88(0.03)$ & $0.89(0.02)$ & 0.15 \\
Pericallosal areas & $0.82(0.04)$ & $0.83(0.04)$ & 0.66 \\
Frontal lobes & $0.81(0.02)$ & $0.82(0.04)$ & 0.50 \\
Temporal lobes & $0.80(0.02)$ & $0.82(0.03)$ & 0.20 \\
Parietal lobes & $0.82(0.05)$ & $0.84(0.03)$ & 0.30 \\
Occipital lobes & $0.78(0.03)$ & $0.78(0.02)$ & 0.95 \\
Internal capsule & $0.87(0.03)$ & $0.87(0.04)$ & 0.91 \\
All white matter areas & $0.84(0.02)$ & $0.85(0.02)$ & 0.36 \\
\hline
\end{tabular}

* Student's $t$ test for non-paired data.

$C$, intervoxel coherence. Corpus callosum $C$ values were obtained by averaging the values of the splenium and genu of the corpus callosum. Pericallosal $\mathrm{C}$ values were obtained by averaging the values of the anterior and posterior pericallosal areas. Internal capsule $C$ values were obtained by averaging the values of genu and capsule $C$ values were obtained
posterior limb of this structure.

disease to be more common than in elderly control subjects. More recently, magnetisation transfer, DW, and DT-MRI studies ${ }^{10-14}{ }_{16}^{16}$ have also shown that, in addition to cortical grey matter damage, ${ }^{16}$ there is a relatively large amount of microscopic white matter pathology in patients with Alzheimer's disease, which goes undetected when using conventional MRI. However, the pathogenetic mechanisms of microscopic white matter damage in Alzheimer's disease are still unclear, including whether Alzheimer's disease microstructural white matter pathology is secondary to or independent of Alzheimer's disease grey matter pathology. Against this background, we performed the present DT-MRI study, in which we quantified water molecular motion and tissue anisotropy of several white matter regions of patients with Alzheimer's disease.

We found that the Alzheimer's disease associated microstructural white matter pathology is not homogeneously distributed, but it rather involves selectively brain white matter regions which are connected with the association cortices (corpus callosum and white matter of the temporal, frontal, and parietal lobes) with a relative sparing of other white matter areas subserving motor (internal capsule) or visual (optic radiations) functions. Our results confirm and extend those of conventional MRI studies, ${ }^{75}$ which showed a notable decrease of the corpus callosum area from patients with Alzheimer's disease compared with age matched healthy subjects. They also agree with the results of a previous DT-MRI study, ${ }^{13}$ which showed that tissue anisotropy of the spenium of the corpus callosum, superior longitudinal fasciculus, and cingulum was reduced in patients with Alzheimer's disease. The preferential location of microstructural abnormalities in white matter regions with a high prevalence of fibre tracts connecting cortical associative areas suggests that wallerian degeneration of white matter fibre tracts secondary to neuronal loss in the associative cortex is a major contributing factor of Alzheimer's disease associated white matter changes. Clearly, other factors (such as ischaemic vascular damage) have the potential to contribute to the DT-MRI changes. However, given the criteria used to select patients for the present study, we think that their role is likely to be minor.

Previous DW DO-12 $^{14}{ }^{15}$ and DT-MRI ${ }^{13}$ studies of white matter in Alzheimer's disease either measured water diffusivity or tissue anisotropy. On the contrary, we measured water diffusivity, FA, and $\mathrm{C}$ of the same white matter areas in an attempt to achieve a better in vivo characterisation of the nature of Alzheimer's disease associated microstructural changes. We found increased $\overline{\mathrm{D}}$ and reduced $\mathrm{FA}$ in the white matter of patients compared to matched healthy volunteers. These findings, which indicate a net loss of barriers restricting water molecular motion and tissue anisotropy of white matter in patients with Alzheimer's disease, agree with histopathological data showing partial loss of myelin, axons, and oligodendrial cells in the white matter. ${ }^{17}$ We also found that $\mathrm{C}$ was not different between patients with Alzheimer's disease and controls in any of the white matter regions studied, including those with reduced FA. This is not the case for patients with multiple sclerosis, in whom a concomitant reduction of FA and $\mathrm{C}$ has been found for posterior pericallosal areas. ${ }^{36}$ Because $\mathrm{C}$ is a measure of intervoxel coherence whereas FA is a measure of intravoxel coherence, the finding of increased FA values in regions with preserved $C$ suggests that the amount of tissue disorganisation of white matter in Alzheimer's disease is relatively mild. Although patients with Alzheimer's disease had lower brain volumes than the healthy volunteers, we think that this has not influenced our results a great deal for two reasons. Firstly, we studied regions located in the white matter away from the brain edges and particular care was used to avoid contamination from both the CSF and the grey matter. Secondly, differences in DT-MRI quantities between patients with Alzheimer's disease and controls were not found in all the white matter regions studied. If partial volume averaging would have been a major contributing factor to the DT-MRI changes found, differences in all the regions studied would have been expected.

The strong correlations found between the MMSE score and white matter $\overline{\mathrm{D}}$ and FA values support the concept that white matter microstructural changes of Alzheimer's disease are likely to contribute to the decline in cognitive function. Although our results are based on a relatively small cohort of patients, the strength of the reported clinical/MRI correlations underpins the importance of white matter pathology in Alzheimer's disease and suggests that quantifying tissue damage of white matter has the potential to improve our ability to monitor Alzheimer's disease evolution. 


\section{ACKNOWLEDGEMENT}

This study was supported by a grant from the Italian Ministry of Health.

\section{Authors' affiliations}

M Bozzali, M Cercignani, M Filippi , Neuroimaging Research Unit Department of Neuroscience, Scientific Institute and University Ospedale San Raffaele, Milan, Italy

A Falini, G Scotti, Department of Neuroradiology, Scientific Institute and University Ospedale San Raffaele

M Franceschi, M Zuffi, Ospedale Santa Maria, Catellanza, Italy G Comi, Clinical Trials Unit, Department of Neuroscience, Scientific Institute and University Ospedale San Raffaele

\section{REFERENCES}

1 Pearson RCA, Esiri MM, Hiorns RW, et al. Anatomical correlates of the distribution of the pathological changes in the neocortex in Alzheimer's disease. Proc Natl Acad Sci USA 1985;82:4531-4.

2 Rogers J, Morrison JH. Quantitative morphology and regional and laminar distributions of senile plaques in Alzheimer's disease. J Neurosci 1985:5:2801-8.

3 Lewis DA, Campbell M, Terry RD, et al. Laminar and regional distributions of neurofibrillary tangles and neuritic plaques in Alzheimer's disease: a quantitative study on visual and auditory cortices. J Neurosci 1987; 7: 1799-808

4 Fox NC, Freeborough PA, Rossor MN. Visualisation and quantification of rates of atrophy in Alzheimer's disease. Lancet 1996:348:94-7.

5 Fama R, Sullivan EV, Shear PK, et al. Selective cortical and hippocampal volume correlates of Mattis dementia rating scale in Alzheimer's disease. Arch Neurol 1997;45:719-28.

6 Foundas AL, Leonard CM, Mahoney SM, et al. Atrophy of the hippocampus, parietal cortex and insula in Alzheimer's disease: a volumetric magnetic resonance imaging study. Neuropsychiatry Neuropsycol Behav Neurol 1997; 10:81-9.

7 Hampel H, Teipel SJ, Alexander G E, et al. Corpus callosum atrophy is a possible indicator of region and cell type specific neuronal degeneration in Alzheimer's disease. Arch Neurol 1998;55:193-8.

8 MacKay S, Meyeroff DJ, Constans JM, et al. Regional gray and white matter metabolite differences in subjects with $A D$, with subcortical ischemic vascular dementia, and eldery controls with ${ }^{1} \mathrm{H}$ magnetic resonance spectroscopic imaging. Arch Neurol 1996:53:167-74.

9 Tedeschi G, Bertolino A, Lundbom N, et al. Cortical and subcortical chemical pathology in Alzheimer's disease as assessed by multislice proton magnetic resonance spectroscopic imaging. Neurology 1996;47:696-704.

10 Hanyu $\mathbf{H}$, Shindo $\mathrm{H}$, Kakizaki $\mathrm{D}$, et al. Increased diffusion in cerebral white matter in Alzheimer's disease. Gerontology 1997;43:343-51.

11 Hanyu H, Asano T, Sakurai H, et al. Diffusion-weighted and magnetization transfer imaging of the corpus callosum in Alzheimer's disease. J Neurol Sci 1999;167:37-44.

12 Sandson TA, Felician O, Edelman RR, et al. Diffusion-weighted magnetic resonance imaging in Alzheimer's disease. Dement Geriatr Cogn Disord 1999;10:166-71.

13 Rose SE, Chen F, Chalk JB, et al. Loss of connectivity in Alzheimer's disease: an evaluation of white matter tract integrity with colour coded MR diffusion tensor imaging. J Neurol Neurosurg Psychiatry 2000;69:528-30.

14 Kantarci K, Jack CR, Xu YC, et al. Mild cognitive impairment and Alzheimer disease: regional diffusivity of water. Radiology 2001;219:101-7.
15 Bozzao A, Floris R, Baviera ME, et al. Diffusion and perfusion MR imaging in cases of Alzheimer's disease: correlations with cortical atrophy and lesion load. ANNR Am J Neuroradiol 2001;22:1030-6.

16 Bozzali M, Franceschi M, Falini A, et al. Quantification of tissue damage in $A D$ using diffusion tensor and magnetization transfer MRI. Neurology 2001;57:1135-7.

17 Brun A, Englund E. A white matter disorder in dementia of the Alzheimer type: a pathoanatomical study. Ann Neurol 1986;19:253-62.

18 Leys D. Pruvo JP, Parent $M$, et al. Could wallerian degeneration contribute to "leuko-araiosis" in subjects free of any vascular disorder? $J$ Neurol Neurosurg Psychiatry 1991;54:46-50

19 Le Bihan D, Turner R, Pekar J, et al. Diffusion and perfusion imaging by gradient sensitization: design, strategy and significance. J Magn Reson Imaging 1991;1:7-8.

20 Basser PJ, Mattiello J, LeBihan D. MR diffusion tensor spectroscopy and imaging. Biophys J 1994;66:259-67.

21 Pierpaoli C, Basser PJ. Towards a quantitative assessment of diffusion anisotropy. Magn Reson Med 1996;36:893-906.

22 Pfefferbaum A, Sullivan EV, Hedehus M, et al. Age related decline in brain white matter anisotropy measured by spatially corrected echo-planar diffusion tensor imaging. Magn Reson Med 2000;44:259-68.

23 McKhann G, Drachman D, Folstein M, et al. Clinical diagnosis of Alzheimer's disease: report of the NINCDS-ADRDA Work Group under the auspicies of Department of Health and Human Services Task Force on Alzheimer's disease. Neurology 1984;39:939-44.

24 Folstein MF, Folstein SE, McHugh PR. Mini-mental state: a practical method for grading the cognitive state of patients for the clinician. $J$ Psychiatr Res 1975;12:189-98.

25 Magni E, Binetti $G$, Bianchetti $A$, et al. Mini-mental state examination: a normative study in Italian elderly population. Eur J Neurol 1996;3:1-5.

26 Jones DK, Horsfield MA, Simmons A. Optimal strategies for measuring diffusion in anisotropic systems by magnetic resonance imaging. Magn Reson Med 1999;42:515-25.

27 Bito Y, Hirata S, Yamamoto E. Optimal gradient factors for ADC measurements [abstract]. Proc Intl Soc Mag Reson Med 1995:2:913.

28 Rovaris $M$, Inglese $M$, van Schijndel RA, et al. Sensitivity and reproducibility of volume change measurements of different brain portions on magnetic resonance imaging in patients with multiple sclerosis. J Neurol 2000;247:960-5.

29 Studholme C, Hill DLG, Hawkes DJ. Automated three-dimensiona registration of magnetic resonance and positron emission tomography brain images by multiresolution optimization of voxel similarity measures. Med Phys 1996;24:25-35.

30 Woods R, Grafton S, Holwes C, et al. Automated image registration I. General methods and intrasubject intramodality validation. J Comput Assist Tomogr 1998;22:139-52.

31 Basser P J, Mattiello J, LeBihan D. Estimation of the effective self-diffusion tensor from the NMR spin echo. Mag Res Series B 1994;103:1-8.

32 Filippi $M$, Cercignani $M$, Inglese $M$, et al. Diffusion tensor magnetic resonance imaging in multiple sclerosis. Neurology 2001;56:304-1 1 .

33 Bowen BC, Barker WW, Loewenstein DA, et al. MR signal abnormalities in memory disorder and dementia. AJNR Am J Neuroradiol 1990;11:283-90.

34 Sheltens $\mathbf{P}$, Barkof F, Algra J, et al. White matter hyperintensities on magnetic resonance in Alzheimer's disease and normal aging. Neurobiol Aging 1990;11:263-4.

35 Vermersch P, Roche J, Hamon $M$, et al. White matter magnetic resonance imaging hyperintensity in Alzheimer's disease: correlations with corpus callosum atrophy. J Neurol 1996;243:231-4.

36 Cercignani M, Bozzali M, lannucci G, et al. Intra-voxel and inter-voxel coherence in patients with multiple sclerosis assessed using diffusing tensor MRI. J Neurol 2002 (in press). 